\title{
Study on Organization Mode in the Enterprises-Led Engineering Application of Considerable Scientific Achievement ---In case of Beijing Enterprises Holdings Maglev Co., Ltd
}

\author{
Shan-shan Sun ${ }^{1,2}$, Feng-ming Wang ${ }^{3}$ \\ ${ }^{1)}$ Harbin Institute of Technology, Harbin, Heilongjiang, China \\ 2) Shanghai Medical Instrumentation College, Yangpu, Shanghai, China \\ ${ }^{3)}$ Beijing Enterprises Holdings Maglev Co., Ltd, Chaoyang, Beijing, China
}

\begin{abstract}
This paper discussed the organization mode of the connotation, characteristics, and structure led by enterprise in the process of scientific and technological achievements, through the analysis of the medium- low speed maglev transportation technology. A company jointing other enterprises established research and development system to independently complete scientific achievement transformation in the form of market mechanism. It is the beneficial trial for considerable scientific achievements. It can accelerate the development of considerable scientific and technological achievements conversion rate.
\end{abstract}

Keywords — maglev transportation, engineering, Beijing S1, organization mode

\section{以企业为主导的重大科技成果工程化过程组织模式初 探---以北京控股磁悬浮技术发展有限公司为例}

\author{
孙珊珊 $^{1,2}$ 王凤鸣 ${ }^{3}$ \\ 1) 哈尔滨工业大学, 哈尔滨, 黑龙江, 中国 \\ 2) 上海医疗器械高等专科学校, 杨浦, 上海, 中国 \\ 3) 北京控股磁悬浮技术发展有限公司, 朝阳, 北京, 中国
}

摘 要 本文以中低速磁浮交通技术工程化为例, 探讨了以企业为主导的重大科技成果工程化的组织模式内涵、特征及网络性的组 织结构。企业以市场机制组建研发体系, 自主进行科研成果工程化研发是重大科研成果工程化的有益尝试, 加速我国重大科技成果转 化率。

关键词 磁浮交通, 工程化, 北京 $\mathrm{S} 1$, 组织模式

\section{1. 引言}

重大科技成果工程化由于技术的复杂性往往表现为周 期长、规模较大、涉及的合作单位多, 是相关产业工业实 力的一个综合体现。重大科技成果工程化过程即包括核心 技术突破、也包括产品可靠性和批量生产等带来的工艺、 标准等问题需要解决。重大科研成果在我国多是由国家组
织实施, 在市场经济发展的今天, 企业以市场为导向的创 新组织组织模式给科研成果的工程化研发带来新的途径。

我国对中低速磁浮交通系统的基础研究开始于上世纪 80 年代, 并取得重大科研成果。如何使这项国际领先的科 研成果不被束之高阁, 需要解决的科研成果工程化难题。

本文将从协同创新组织模式的视角, 以北控磁浮公司

国家科技支撑计划项目支持 (资助号：2012BAG07B01) 
成功完成该项技术的工程化开发并在北京轨道交通 $\mathrm{S} 1$ 线得 到工程应用为范例, 研究工程化研发过程中采取的新型协 同创新组织模式的特点和发挥作用的机理等, 以期提高我 国重大科研成果转化率提供经验借鉴和模式参考。

\section{2. 重大科研成果工程化组织模式}

重大科技成果按照其用途可分为: 国防科技类、重大 装备类、新能源、新材料; 交通运输类等, 其工程化过程 普 遍具有以下特征: 技术复杂系统性、高 风险性、涉及众多 合作单位、工程化周期长、组织协调难度大、需要大量资 金持续的支持。重大科研成果工程化过程的特征决定了其 组织过程的复杂性和协同性特点, 往往由国家层面组织工 程化研发、生产和实施。国家一般设立项目的办公室, 成 员为相关的国家部委和国家级组织机构, 负责统筹研发、 设计、生产等环节的协调工作，以及各块工作的资金分配; 在该项目办公室内下设若干项目组, 各项目组具体负责科 研、生产、建设等工作, 向相应科研机构、企业下达任务 [1-4]。

高铁列车工程化就是典型的国家主导的重大科研成果 的工程化项目。参见图 1 (以京沪高铁为例)。2007 年国务 院成立 “京沪高速铁路建设领导小组” 由国务院副总理担 任组长, 国务院副秘书长、发改委主任、铁道部部长任副 组长, 科技部等 13 个部委、工程院、银监会、京沪高铁沿 途 7 省市领导任工作组成员。京沪高速铁路领导小组负责 跨行业、跨部门的科研、设计、生产和建设中的组织和协 调工作, 铁道部负责具体工作。铁道部把京沪高铁工程化 实施工作分成若干项目, 由铁道部科技司等各司局负责科 研攻关、产业化研发、工程实施等, 为投融资需要成立 “京 沪高铁股份公司”, 承接铁道部各司局下达的各项任务, 并 把任务下发给相关科研、设计、生产和施工单位 [5-6]。这 种层级制的组织模式, 由国家下拨经费, 负责跨部门、跨 行业的技术攻关和建设施工, 每级单位只对上级负责, 避 免了同级单位及跨行业、跨部门合作单位之间的扯皮, 参 与企业的市场风险很小。

国家组织的重大科研成果工程化组织模式构成层级 式, 对于参与企业采取计划经济的管理模式, 向企业下达 指令。但是这种模式的灵活性不足, 企业根据市场需求牵 头组织的重大科研成果工程化成为其有益补充。譬如, 能 够影响我国轨道交通架构的中低速磁浮交通技术, 是在科 技部、北京市等政府部门的持续支持下取得的国际领先重 大科研成果, 但是其工程化研发不是由国家而是由企业在 市场原则下组织完成的。

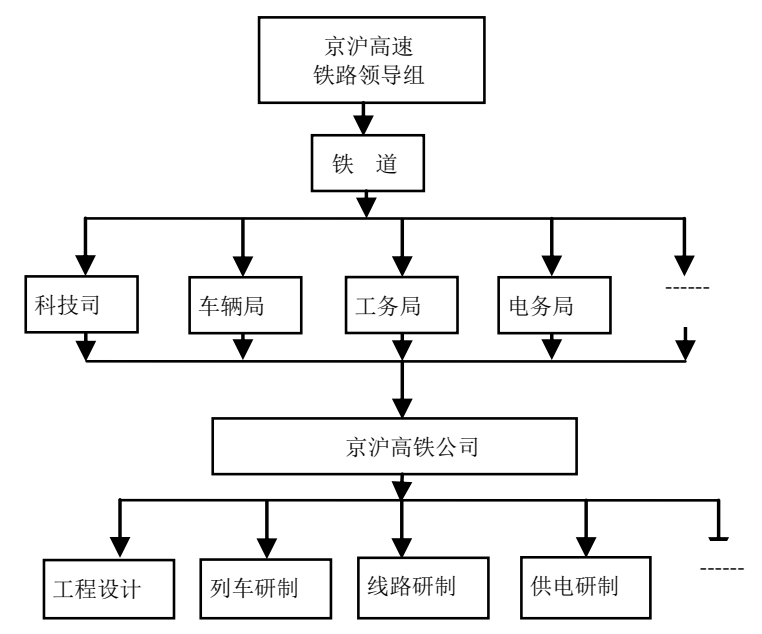

图 1 京沪高铁组织模式

\section{3. 中低速磁浮交通技术的工程化网络组织模式}

上世纪末, 北控集团为解决北京八达岭景区交通和景 观协调问题, 把长城脚下的停车场向外迁移约 2 公里, 为 解决交通接驳问题, 在广泛论证现有各种交通运输方式之 后, 决定采用中低速磁浮交通系统。但是这项技术在当时 还处于实验室的研究成果阶段, 仅仅只做了原理样机, 北 控集团敏锐的扑捉到中低速磁浮交通技术优势, 决定在国 防科技大学前期研究成果基础上, 推进中低速磁浮交通技 术工程化开发, 开始了以解决八达岭景区交通问题为初始 目标中低速磁浮交通技术工程化开发

中低速磁浮技术工程化采取了北控磁浮公司牵头的网 络组织模式, 北控集团与科研成果的发明人国防科大合作, 联合国内铁路、航空、汽车、冶金等领域十几个在行业内 具有优势的企业组成中低速磁浮交通技术工程化开发体 系, 历时十年完成了通常由政府牵头的重大科研成果工程 化研发, 并成功在北京轨道交通 S1 线得到应用。

\section{1 企业牵头的网络型协同创新组织模式内涵}

中低速磁浮交通系统利用受控制电磁力把车辆悬浮在 轨道上取代了传统地铁的轮轨关系。整个系统划分为车辆、 线路轨道、信号和埽引供电等四个大系统组成。构成中低 速磁浮交通系统的单体设备、产品都是关键技术、子系统、 系统集成而成, 在技术研发过程中各子系统的技术相互匹 配性直接影响中低速磁浮技术的系统综合性能, 因而在工 程化过程中技术的协同研发是中低速磁浮交通技术工程化 过程中最重要的特征。技术的协同性要求建立工程化过程 中要建立一个高效的组织机构和合理的组织机制来有效地 组织各级技术系统的研发和生产。中低速磁浮交通技术协 同创新的组织模式特点 [7-8]: 1) 整体性: 以产学研联盟形 式把科研院所为主体的科研成果原始创新主体和以企业为 
主体的生产技术创新紧密联合在一起, 弥合了科研机构和 企业之间缺乏有效沟通的弊端; 2) 以牵头企业为主导的网 络型组织结构: 北控集团作为中低速磁浮交通技术工程化 的组织单位, 牵头组织并出部分科研启动资金, 各协作单 位进行资金配套共同进行中低速磁浮交通技术的工程化研 发。北控集团和各合作单位是网络型的组织结构。

\section{2 中低速磁浮交通技术工程化网络组织模式}

北控集团与科研成果的发明人国防科大合作, 组织联 合我国铁路、航空、汽车、冶金等相关领域最具优势的研 究、设计、生产和建设单位, 以实现自主知识产权、国产 化、世界领先的中低速磁浮交通产业为目标，基于 “共同 投入，风险共担、利益共享” 原则组建了工程化开发体系。 经过十年艰苦努力共同攻克了中低速磁浮交通技术工程化 过程中专有产品研发、批量生产工艺、质量控制标准等难 题, 完成了这项科研成果的工程化研发并取得实际工程应 用。这次的工程化研发成功, 代表着一种以企业为主体的 协同创新组织模式的诞生, 这种协同创新组织模式将会为 我国科研成果转化率的提高提供有益参考和借鉴。

依靠共同的理想和市场目标, 北控磁浮公司联合众多 合作单位组织了十几倍于自身资金共同投入，成功完成了 中低速磁浮交通技术的工程化研发。如图 2 所示, 北控磁 浮公司作为工程化活动的发起者, 负责项目的组织协调, 科研成果的发明人国防科大负责技术集成, 列车集成和零 部件研制由唐山轨道客车有限公司、株洲南车时代电气股 份有限公司、上海飞机公司承担, 轨道设备所属道岔和 $\mathrm{F}$ 型轨道由宝鸡桥梁厂和莱芜钢铁集团完成, 信号系统和供 电系统的研制由中国铁道通信信号集团公司和中铁电气化 局集团公司承担, 工程设计和施工由铁道第三勘察设计院 集团有限公司和中铁六局等单位承担。

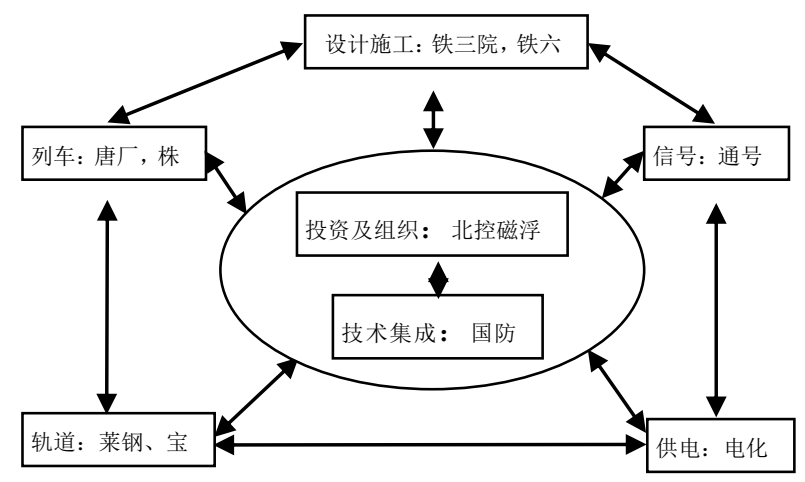

图 2 中低速磁浮技术工程化组织模式

作为项目的组织协调方, 北控磁浮公司与各方签订合 作协议, 根据合作内容、产品性质和市场预期给予不同额
度的资金支持，但是主要资金来源由各企业自行配套解决。 但是在开发新产品的过程中北控磁浮公司和国防科大提供 技术支持和前期科研成果。

作为系统集成和科研成果的发明人国防科大的地位比 较特殊, 学校是科研的主力军, 工程化研发离不开原始科 研成果的技术支持。但是工程化进程需要的大量资金投入 需要由企业承担, 成果发明人以技术优势参与到这个过程。

另一方面, 科研成果的工程化研发以企业为主导更加 有效。在中低速磁浮交通技术这项科研成果的工程化过程 中, 组织人北控磁浮公司发挥企业自身工程组织和市场推 广特长协调参研单位各方利益和收益预期; 科研成果发明 人国防科大借助组织方把新技术成果贯彻到各工程化体系 单位的产品研制中; 工程化合作体系各参研单位借助科研 成果涵盖的新技术，利用项目组织方的启动资金带动企业 自身更大投入, 为企业开发了新产品和新市场。目前这项 科研成果的工程化开发取得成功, 技术在实际工程建设中 得到应用, 各方预期得到实现。

\section{参考文献(References)}

[1] Cun-jin, Li, A preliminary study on the system integration organization model of large-scale science and technology engineering Journal of Science and Technology Management Research, 2013, 15.

[2] Jia-xi, Ma, Study on the model of enterprise technological innovation organization pattern choice Journal of Science of Science and Management of S \& T, 2008,5.

[3] Zhi-gang, Feng, Research for technology innovation enterprise integration patterns Journal of Science and Technology Research, 2004,4.

[4] Wei, Fang, Research on the organization mode of the enterprise technology innovation of multiple projects Journal of Forum Science and Technology in China, 2006, 5.

[5] Fan-zhu Kong, Research on knowledge management of enterprise integrated innovation and cooperation innovation interaction mechanism Journal of Science \& Technology Progress and Policy, 2011, 8.

[6] Ki-Seok Kwon, Synergy or separation mode: the relationship between the academic research and the knowledge-transfer activities of Korean academics, Scientometrics, January 2012.

[7] Jing-wen Han, Internal Mechanism Research on Synergy of Enterprise Network Organizations Based on Value Structure, The 19th International Conference on Industrial Engineering and Engineering Management, 2013.

[8] Thomas Bolli, Competition and R\&D cooperation with universities and competitors, The Journal of Technology Transfer, December 2013. 